\title{
La comunicación como factor clave en la experiencia del consumidor para la fijación del precio
}

communication as a Key Factor in the Consumer Experience for Price Fixing

Ariana Paola Herrera Pérez

Doctoranda en Comunicación Audiovisual, Publicidad y RR.PP

Universidad Complutense (Madrid, España) arherr01@ucm.es
Herrera Pérez, A.P. (2020)

La comunicación como factor clave en la experiencia del consumidor para la fijación del precio

Revista Internacional de Investigación en Comunicación aDResearch ESIC. № 21 Vol 21

Primer semestre, enero-junio 2020 · Págs. 84 a 97

https://doi.org/10.7263/adresic-021-05 


\section{Clasificación JEL: M31 y L83 Palabras clave:} Comunicación, marketing experiencial, marketing sensorial, fijación de precios
Objetivo: El objetivo de la presente investigación es analizar la comunicación como uno de los generadores de experiencia del consumidor y su influencia en la fijación del precio. El mecanismo tradicional que utilizan empresas del sector de restauración para establecer el precio de venta al público se basa en los costos, dejando por fuera el valor percibido por el cliente a nivel experiencial.

Diseño/metodología: La metodología de investigación abarca dos fases. La primera fase metodológica requiere una exploración bibliográfica analítica-sintética, que explica el contexto y el estado de la cuestión mediante una recopilación bibliográfica de autores que corroboran la relación existente entre la comunicación, estrategia experiencial, precio y comportamiento del usuario. La segunda fase de la investigación es de carácter deductivo, para la recolección de datos se emplea el muestreo no probabilístico de conveniencia. Se aplica un servicio personalizado y posterior una encuesta a quince profesionales en sus respectivas oficinas en Ecuador.

Resultados: Los resultados muestran que los clientes estarían dispuestos a pagar cinco veces más por un servicio personalizado y diferenciador, por ende, un factor clave en el proceso de servicio es la comunicación bidireccional entre el prestador del servicio y el cliente. Mediante esta implementación se comprobó que el individuo realmente paga por lo que un producto o servicio representa a nivel experiencial, y queda en segundo plano, lo racional.

Limitaciones/implicaciones: La implicación de los resultados expuestos es de carácter empresarial con enfoque a la toma de decisiones respecto al precio. No obstante, los datos recolectados mediante un servicio de restauración no permiten la utilización generalizada de los resultados por su naturaleza.

Originalidad/contribución: La investigación se caracteriza por el valioso aporte inédito al estudio de la comunicación y la experiencia del consumidor como un mecanismo de fijación de precios desde una perspectiva holística, aislando los costos como eje principal de cálculo y toma como referente esencial la experiencia del usuario.

\section{ABSTRACT}

\section{JEL Classification: M31 y L83 Key words:} Communication, experiential marketing, sensory marketing, pricing
Purpose: The objective of the current research is to analyze the communication as one of the drivers of customer experience and its influence in price selection. The traditional mechanism that businesses of the catering sector use to establish the sales price for the public it is based only in costs, leaving aside the perceived value of client at a perceived level.

Design/methodology: The methodology for this research is divided into two phases. The first methodological phase requires an analytical-synthetic exploration of current bibliography, that explains the context and the state of the art for the topic at hand through a bibliographic review of several authors that corroborate the relationship between the communication, perceived strategy, price setting and user behavior. The second phase of the research is of a deductive nature. For the gathering of the data, a non-probabilistic convenience sampling is applied; this has been done through the implementation of a personalized drinks delivery service, applied to professionals in their respective offices in Ecuador, who after intake of the beverages a survey has been conducted.

Findings: The results show that the customers would be willing to pay five times more for a personalized and differentiated service, as such, a key factor identified in the service process is the bidirectional communication between the service provider and the client. Through this implementation it has been corroborated that the individual is really paying for what the product or service represents at a perceived level, relegating the rational part to a second level.

Research limitations/implications: The implication of the presented results is of a managerial nature, with a focus in the decision process of price selection. Nevertheless, the data collected are in regards to a single type of catering service, and thus, do not allow for a generalization of the results obtained.

Originality/value: The present research it's characterized by its valuable and unpublished study of the relationship between communication and customer experience as a mechanism of price selection from a holistic perspective, isolating costs as the main drivers of the calculation and taking the customer experience as an important variable. 


\section{Introducción}

La comunicación es uno de los principales proveedores de experiencia y su impacto es directo sobre los módulos experienciales estratégicos. La comunicación bidireccional entre el prestador del servicio y el cliente es clave en la generación de experiencia del consumidor. Aunque la comunicación es parte de la cotidianidad, se ha convertido en lo último en la que el individuo reflexiona (Vidales, 2015). Porque la comunicación es realmente efectiva cuando el receptor logra entender el mensaje que le fue transmitido (Ferrés y Masanet, 2017). Comunicarse se convierte en una forma de promover la nostalgia emocional hacia la marca, creando sentimiento de apego y afinidad resultado de la cercanía con el cliente (Wen, Qin and Liu, 2019). A través del tiempo, la comunicación directa ha ido perdiendo su transcendencia a nivel empresarial. El desarrollo de las nuevas tecnologías ha desplazado a la comunicación presencial en los clientes internos de una organización (Victoria y Herrero 2020), generando una brecha en la calidez del servicio hacían el cliente externo, desapego hacia las marcas y dificultando la fijación de precios.

Diferentes autores enfatizan que el uso excesivo del teléfono móvil puede causar el fenómeno del «temor de perderse de experiencias» (Santana, Gómez y García, 2019). Por estas problemáticas socioculturales, la experiencia del consumidor para las medianas y grandes empresas del sector de la restauración se ha convertido en un punto esencial de su política de marketing, por ello, es vital diferenciar su servicio de la competencia existente dentro o fuera de su nicho de mercado. Esto marca el nivel de competencia y la tasa de rentabilidad que les ayude a elevar sus ingresos. La curva de la experiencia está basada en las perspectivas de un cliente. Por otro lado, la fijación de un precio está conectada con el valor sentimental, su calidad, fácil acceso o por la seguridad que este genera. Este estudio recopila información relevante respecto a la importancia que tiene la comunicación en la experiencia de los consumidores a la hora de establecer un precio.

Los beneficios de lo ofertado ya no son suficientes para incentivar una compra. Es necesario influir directamente en el usuario, promoviendo una experiencia que conlleve a la creación de la credibilidad de la marca y esto solo se logra con excelencia al momento de la recepción del servicio (Nayeem, Murshed y Dwivedi, 2019). En consecuencia, existe la necesidad de ofrecer productos y servicios atractivos de valor basado en aspectos emocionales vivenciales.

El profesor Bernd Schmitt fue uno de los primeros en introducir el concepto de marketing experiencial en los años 90 del siglo pasado. El experto lo vio como una extensión del marketing tradicional (Schmitt, 1999). Además, integró varios métodos, conceptos y medios utilizados actualmente en productos y campañas de marketing, que involucran o atraen al consumidor por las experiencias. Es aquí cuando nace la necesidad de crear experiencias holísticas para los clientes y así involucrar un valor sentimental. Se encuentra en la constante búsqueda de generar una experiencia única para los clientes e inducir su compra. La intención es hacerles sentir, pensar, actuar y relacionarse.

Estas estrategias son útiles para que las empresas creen una experiencia agradable para los clientes, aumentar los valores del producto y mejorar la satisfacción del cliente y lealtad hacia la marca. Schmitt (1999) lo define como «un proceso de creación de experiencias». Las empresas diariamente se encuentran en una guerra de precios y esta es una oportunidad para crear valor agregado y reducir acciones con efectos a 
corto plazo. Para ello, una propuesta de valor diferenciadora requiere una alta dosis de creatividad, en especial si se estimulan los sentidos (Singhal y Khare, 2015).

Es aquí donde destaca lo innovador de esta investigación: la mayoría de mecanismos de fijación de precios al consumidor final se basan en los costos, margen de contribución, demanda, rentabilidad sobre la inversión y afines, pero respecto a la experiencia del consumidor como variable determinante son escasas las metodologías reales empleadas por las empresas. Se pretende demostrar que, a pesar de invertir pequeños costos de producción, se puede cobrar un valor mayor por el servicio prestado, mediante la generación de una vivencia o experiencia fuera de lo común. La evolución del mundo conlleva una vida agitada y con poca disponibilidad de tiempo para que el ser humano se atienda y se dé gustos en servicios personalizados. Se ha identificado esta necesidad, que será el vínculo de conexión para la experimentación servicio vs precio.

De acuerdo a lo anterior, el objetivo general de la presente investigación es analizar cómo la comunicación influye en la experiencia del consumidor y consigo, su influencia en la fijación del precio. A partir de este objetivo general se detallan los siguientes objetivos específicos: investigar acerca de los módulos de experienciales estratégicos y el consumidor; estudiar la comunicación como un proveedor de experiencia y su influencia en la fijación de precios; analizar la importancia de la estimulación sensorial en el proceso de compra mediante la implementación experimental de un servicio; comprobar si una experiencia diferenciadora define el precio de un producto o servicio en el proceso de compra, como un elemento determinante a tener en consideración a nivel empresarial; entender si una experiencia positiva en la percepción de valor económico del consumidor es mayor que los costos implícitos en el servicio.

\section{Marco teórico}

\subsection{Marketing experiencial herramienta eficaz en la generación de una propuesta de valor diferenciadora}

En un mundo globalizado y ante un perfil de consumidor exigente, la diferenciación de la oferta se hace cada vez más difícil. Para lograrlo se debe alcanzar la co-creación de la experiencia entre las partes involucradas a nivel interno y externo, alcanzando así intereses en común (Erhardt, Martin y Chan, 2019). La satisfacción del cliente ya no es sencilla y para diferenciarse se necesitan experiencias. En este medio, surge con fuerza el Marketing Experiencial. Es importante tratar de seducir a los consumidores mediante la optimización de la «experiencia vivida». El empleo adecuado de la tecnología en la comunicación permite una conexión cercana con el consumidor y a su vez la creación de valor en la experiencia (Zhang et al., 2018). Es necesario evitar la incertidumbre experiencial con el objetivo de fomentar confianza y fidelización a través del tiempo (Wu, Chen y Cheng, 2019). La auténtica comunicación se da cuando somos capaces de expresar sentimientos y emociones a los demás. Por esta razón, se requiere el empleo de acciones de contacto directo entre la marca y el cliente; como un mecanismo de interacción en el área de alimentos y bebidas son las salas de degustaciones, las cuales sirven como un generador de conciencia de las bondades de los productos ofertados (Cuellar, Eyler y Fanti, 2015).

Para el caso de las ventas online la generación de confianza es un camino más complejo, se requiere notoriedad, imagen y calidad en el servicio (Higueras, Alard y Mercado, 2016). El marketing experiencial, según Balan (2015): «Aplica dentro 
del concepto de la psicología ya que ve a los consumidores como personas racionales y emocionales». Además, la emoción es el punto estratégico donde gestiona la experiencia. Y se promueve a los gerentes a incrementar estrategias en el diseño de experiencias (Castañeda, Del Valle y Martínez, 2018). La comunicación es la encargada de entregar el contenido y mensaje emocional correcto, en el lugar oportuno a los clientes adecuados. El marketing experiencial abarca cinco tipos de experiencias o módulos experienciales estratégicos: sensaciones, sentimientos, pensamientos, actuaciones y relaciones que juntas forman la experiencia en general.

\subsection{Módulos experienciales estratégicos - MEE}

El marketing sensorial es utilizado para estimular los sentidos de los consumidores mediante diversas atmósferas. De acuerdo a la investigación realizada por Lindstrom (2015), hay una conexión entre el consumidor y la marca a través de la interacción de los cinco sentidos. Con el objetivo que las emociones dominen el pensamiento racional, el marketing sensorial les brinda a las compañías oportunidades y conexiones para diferenciarse, a través de estrategias que vinculan los sentimientos (Hulten, 2015; Kumar, 2016). El marketing sensorial y experiencial convergen en una estrategia con la finalidad de llevar al consumidor a un ambiente más llamativo en la atmosfera en el que se desarrollan las actividades de los clientes (Villanueva y García, 2017). Las sensaciones tienen la apertura de cinco sentidos: vista, oído, olfato, gusto y tacto. El objetivo es la estimulación sensorial.

Para el marketing de sensaciones los sentidos más importantes son la vista y el oído, por su rápida comprensión para crear una experiencia mediante los colores y sonidos. En la forma en que el ser humano procesa las imágenes el sistema mental se implica en el procesamiento de la estimulación experimental (Le, Scott y Lohmann, 2019). Los colores están presentes en cualquier lugar y producen un impacto directo. Pueden expresar ideas, sentimientos y el estado de ánimo (Avendraño et al., 2015).

Una estrategia empleada para influir en los sentidos es el visual merchandising que facilita a las empresas generar reconocimiento de marca y a su vez, posicionamiento de manera coherente y efectiva. Igualmente, conduce a los consumidores a simplificar las decisiones de compra (Llovet, 2018). Refleja el estilo y el carácter de una empresa y permite diferenciarse del resto, logrando su identidad corporativa. En general, son utilizados dependiendo las características físicas del producto o por la clasificación del mercado. Para el mercado, según su imagen corporativa, existen las siguientes clasificaciones:

Alimentos: naranja, amarillo, rojo, verde y café. Ej. Starbucks: verde y café

McDonald's: amarillo y rojo.

Salud: blanco, verde y azul.

Ej. Farmacia cruz azul - Ecuador: azul y blanco. Importancia y prestigio: violeta, rojo, blanco, dorado, negro y plata.

Ej. Microsoft: negro y blanco.

Exclusivo: colores modernos y excéntricos, colores de moda.

Ej. Zara: negro y plata.

Dior: colores de moda.

En el caso del oído, la música es un excelente medio de comunicación. Influye en las emociones y comportamiento de los clientes. La música debe ser elegida cuidadosamente, ya que la escuchamos prácticamente en todo momento y todo lugar. La música incide en las respuestas emocionales del cliente (Suárez y García, 2012). 
Es así como la experiencia del cliente desplaza al producto y prevalece la importancia del ambiente donde se desarrolla el consumo (Manzano et al., 2012). Esta es la búsqueda de generar publicidad en el punto de venta, que ha generado que las marcas desarrollen e innoven en estrategias para estimular al cliente (Robles, 2009). Aunque es vital que haya coherencia entre los mensajes auditivos que se entregan y la experiencia. Hay tres objetivos que plantea Bernd Schmitt (2000). El primero son las sensaciones como elemento diferenciador; el segundo, como motivador; y el último, como proveedor de valor.

1. Diferenciador: La diferenciación puede atraer de una forma inusual y espacial de apreciar un producto o servicio diferente, de manera que tenga una mayor valoración por parte de los consumidores. Por esta razón se requiere estimular los sentidos mediante la creación de la atracción sensorial. Un ejemplo podemos encontrarlo en los autos Jaguar. No solo brindan un alto rendimiento, carrocería de lujo y originales diseños, sino que entregan a sus dueños la experiencia de tener un vehículo único.

2. Como motivador: Para promover la compra de los productos y servicios de una compañía es necesario alcanzar el nivel perfecto de provocación. Es importante entender aquellas necesidades aspiracionales del individuo, que a simple vista no se ven.

3. Sensaciones como proveedor de valor al cliente: Esto no es más que entregar beneficios adicionales como estímulos y proceso (visual, auditiva, olfativa y táctil) para transmitir información. Desde que amanece, el ser humano es impactado por numerosas estrategias de comunicación publicitaria. Starbucks emplea el marketing de sensacio- nes, enfocado al consumo y pone al cliente a disfrutar en un ambiente hogareño. Transmite una vivencia a través del olor, del sabor y del sonido. El diseño y la decoración de sus locales contribuyen a crear un ambiente cómodo y acogedor, como si estuvieran en casa. Con esto logra crear una propuesta de valor a sus clientes.

En este proceso es necesario rescatar la importancia del marketing de sentimientos, que genera un lazo afectivo. Es vital crear sentimientos hacia la empresa durante la experiencia de consumo. El objetivo es la vinculación afectiva hacia la marca a través de fuertes emociones de alegría y orgullo. En la actualidad, existe un mecanismo de humanizar la marca y es a través de influencers, quienes mediante una vivencia en primera persona, comparten su experiencia. Se los conoce también como «micro celebridades» por ser personas influyentes en social media pero no en medios masivos (Ruiz Gómez, 2019).

Es una clave para fomentar la lealtad de marca. La interacción o el hecho de estar cara a cara son la causa más importante de sentimientos encontrados entre los usuarios. Las personas tienen sentimientos muy encontrados no solo entre sí, sino también hacia productos o servicios. Un claro ejemplo es Disney. La propuesta de valor de la marca es «Un mundo mágico». Está compuesto por sus increíbles parques temáticos y películas. Para que las estrategias de sentimientos tengan éxito deben transmitir un mensaje claro al consumidor. Hay que buscar el camino correcto para que el cliente logre percibir, de manera oportuna, lo que la empresa busca comunicar.

El marketing de pensamientos cumple la función de animar al cliente que piense creativamente. Según Bernd Schmitt (2000): «La receta para el éxito de una campaña de pensamientos depende de tres ingredientes clave: el factor sorpresa, la 
intriga y la provocación». El factor sorpresa: Es esencial para cautivar al cliente y vincularlo con el pensamiento creativo. La sorpresa se da cuando los clientes reciben más de lo que piden. La intriga: Transciende del factor sorpresa, despierta la curiosidad de los clientes. La provocación: Tiene el poder de generar un debate, crear tendencia, controversia o escandalizar. Cuando una campaña logra juntar estos tres elementos, crea parte de una experiencia total y permite que el consumidor se sienta atraído por el tipo de mensajes creativos que recibe (Schmitt, 2000).

Las estrategias de actuación cumplen un papel protagonista en la comunicación de la campaña. Están destinadas a crear experiencias relacionadas con el cuerpo físico. El marketing tradicional ignora la experiencia de actuación. El marketing de actuaciones y sus dos puntos fuertes crean experiencias corporales y estilo de vida. Un claro ejemplo son las estrellas de cine o deportistas que aparecen en anuncios, televisión, páginas web, redes sociales, etc. Un porcentaje de la comunidad de seguidores tienden a imitarlos y cambiar su formato de vida, especialmente la de experiencias del ejercicio físico. Día a día, generan contenido para los consumidores mostrándoles alternativas de hacer las cosas que van desde la alimentación hasta lo espiritual. Los bloggers de viajes también marcan tendencias con fotos increíbles posando en lugares, convirtiéndolos en destinos por las caracterizaciones fotográficas que logran y las bondades que muestran de las propiedades y sus servicios.

\subsection{Fijación de precios}

Con el paso del tiempo, las empresas se han vuelto más competitivas y han establecido importantes estrategias en diferenciación, comunicación, marketing y sobre todo, en precios. Existen factores, tanto internos como externos, que influyen en la decisión de fijar precios. Estos pueden variar de- pendiendo de las políticas que posee la compañía. Uno de los autores más influyentes respecto a las tácticas de fijación de precios es Carlos Sánchez, con su libro Administración y estrategias de precios, publicado en México en el año 2012. El autor hace referencia a ochos tipos de tácticas y una de ellas es la de precios basados en la curva de experiencia.

Para establecer precios, un vendedor debe conocer a fondo sus productos o servicios y para qué tipo de clientes fueron elaborados y qué demanda pretenden satisfacer. Se analizan las características que poseen, para establecer un valor acorde a la calidad del bien ofrecido y garantizar una utilidad por encima de los costos. La gestión de las marcas prevé el mejor posicionamiento en la comunicación social a través de mensajes con creatividad, que persiguen la conexión emocional con los grupos (Benavides, 2017).

Hoyer, MacInnis y Pieters (2015) indican que: «El precio es una de las primeras características o factores que influyen en el comportamiento del consumidor y su decisión de compra». Además, se reconoce el precio como uno de los motivos de análisis que conlleva a desistir o realizar el proceso de compra. Así lo confirma la investigación realizada por Raiteri (2016), quien rescata el comportamiento del consumidor como «el proceso de decisión y la actividad física para buscar, evaluar y adquirir bienes y servicios para satisfacer las necesidades». De acuerdo a la misma autora, el comportamiento del consumidor es la forma en cómo un cliente siente la necesidad de satisfacer sus deseos aspiracionales. El cliente investiga, evaluar las alternativas y así determina la compra (Raiteri, 2016).

Pero, ¿cómo influye la fijación de precios en el comportamiento del consumidor? Fijar un precio debe ser un proceso que pueda garantizar una ganancia económica para el vendedor y una satisfacción emocional para el comprador. La 
curva de la experiencia hace referencia a cómo el consumidor se siente conectado emocionalmente con la marca y el sentimiento que genera para su vida. La lealtad que genera en el cliente debe ir más allá del proceso de compra, debe trascender en el uso y frecuencia de adquisición (Obiegbu, Larsen y Ellis, 2019).

La compañía Columna Brand Strategy \& Colors (2017) señala que «la experiencia del consumidor comienza a superar al precio y al producto como diferenciadores de la marca». Esto se refiere a las marcas, como bienes tangibles e intangibles. Las empresas deben ir aún más lejos, innovando ideas para crear una experiencia ideal para sus clientes. Relacionar al cliente con el tipo de bien y servicio ofrecido para fidelizarlo y hacerlo sentir indispensable.

Las experiencias para los clientes no solo se basan en el packaging, la marca o calidad. Se enfocan en el sentimiento de agrado, de ser valioso y de ser bien atendido. En la actualidad, las opiniones y valoraciones de los clientes están disponibles a un clic, mediante la huella digital que registramos en línea (Herrera, 2019). Desde el momento en que el cliente entra al local, el 50\% de compra ya está hecha y lo demás depende únicamente del vendedor. Raiteri (2016) también matiza: «Cuando un vendedor se acerca al comprador y le brinda su tiempo no solo para hablarle del producto que se ofrece, lo hace sentir al cliente como un miembro más de esa organización». Eso ya se lo cataloga como experiencia para el cliente y hay que mantener ese nivel de satisfacción.

El ser humano, por su naturaleza, busca comunicarse, ser sociable y formar amistades. Para un vendedor, uno de sus objetivos debe ser convertirse en sus fieles acompañantes durante el recorrido de compra o pasillo, estando atento, escuchando y dando recomendaciones que faciliten su decisión. Una experiencia se basa en algo pasado, que marcó cierto punto de su vida, lo cual provocará en ellos el deseo de volver a vivirlos. Carl Gustav Jung (2016) establece que: «La experiencia del cliente, es el producto de las percepciones de un cliente después de interactuar racional, física, emocional y/o psicológicamente con cualquier parte de una organización».

Es imprescindible generar recuerdos que impulsan el deseo de compra, fidelización y percepción positiva del precio. Ya no lo verá como algo costoso, sino que entenderá por qué su valor es elevado y comprenderá que vale la pena pagar por algo diferente.

A esto se lo conoce como estrategia de experiencia diferenciadora, la cual consiste en generar más valor y centrarse en lo emocional.

\subsection{Experiencia en el precio}

Todo consumidor busca satisfacer sus necesidades. Algunas con características racionales y otras, emocionales. Los gerentes buscan generar compromiso hacia las marcas por parte de los clientes, mediante la satisfacción (Keiningham et al., 2017). Cada individuo tiene requerimientos específicos alineados a distintas clases sociales y en base a éstas, están distribuidos los bienes y servicios. Así, se considera conveniente seleccionar los públicos y encontrar canales alternativos de comunicación que generen valor añadido a través de nuevos formatos (Juaneda, Olarte y Pérez, 2018).

Un precio depende del valor percibido por el consumidor. Es necesaria la generación de experiencia holísticas que engloben varios elementos experienciales y permitan una conexión con el servicio a largo plazo (Anninou y Foxall, 2019). Un bien de precio económico es accesible a todo público. Sin embargo, no siempre representa calidad y durabilidad. Por otro lado, un precio alto genera confianza, calidad, innovación y diferenciación. Un ejemplo de fijación de precios por experiencia son 
los hoteles: mientras la valoración en las comunidades de viajeros sea la mejor, el ingreso de los hoteles es mayor por la relación directa que guardan entre sí (Majó, Moya y Vall-Llosera, 2018).

\section{Metodología}

La presente investigación profundiza en datos bibliográficos y estadísticos de varios autores, que ratifican la influencia de la experiencia del consumidor en la fijación de los precios. La primera fase metodológica requiere una exploración bibliográfica analítica-sintética, que explica el contexto y el estado del arte. En esta fase se analiza la comunicación, marketing experiencial y la fijación de precios. Esta información, posteriormente, se enlaza con la segunda fase de la investigación que es de carácter deductivo. Para la recolección de datos se emplea el muestreo no probabilístico de conveniencia, a través de la aplicación de un servicio personalizado y posterior una encuesta a quince profesionales en sus respectivas oficinas en Ecuador. Los datos obtenidos de la encuesta se tabularon mediante Excel, con el fin de expresar la estadística mediante gráficas que permitan la lectura práctica y sencilla. Las preguntas realiza- das están orientadas a responder los objetivos de estudio, alineadas a servicio, experiencia, precio y valor percibido. Para el desarrollo de la actividad, se emplea un servicio a domicilio y una carta de bebidas sin alcohol, servido a colaboradores en sus oficinas en Ecuador. Los costos totales por cada té sumaron 0,20 centavos de dólar y el precio de venta fue de 1,50 dólares. El fin es obtener una conclusión asertiva del impacto de una experiencia positiva y diferente en el precio; y responder los objetivos de estudio de la presente investigación.

\section{Resultados}

Para la generación de los resultados se creó una experiencia personalizada, mediante la estimulación sensorial a 15 colaboradores de diversas oficinas. A continuación, se detallan los datos obtenidos: En la gráfica número 1 se destaca que un 93,3\% de los encuestados ha posicionado el servicio con una valoración de cinco estrellas, mientras que el 6,7\% lo valora con cuatro estrellas. Con estos resultados podemos demostrar que todos los clientes que recibieron el servicio quedaron satisfechos con la experiencia vivida.

\section{Gráfica 1 - Diseño de barras, representación porcentual del precio con respecto al servicio ofrecido al grupo de clientes seleccionados}

Calificación del servico brindado

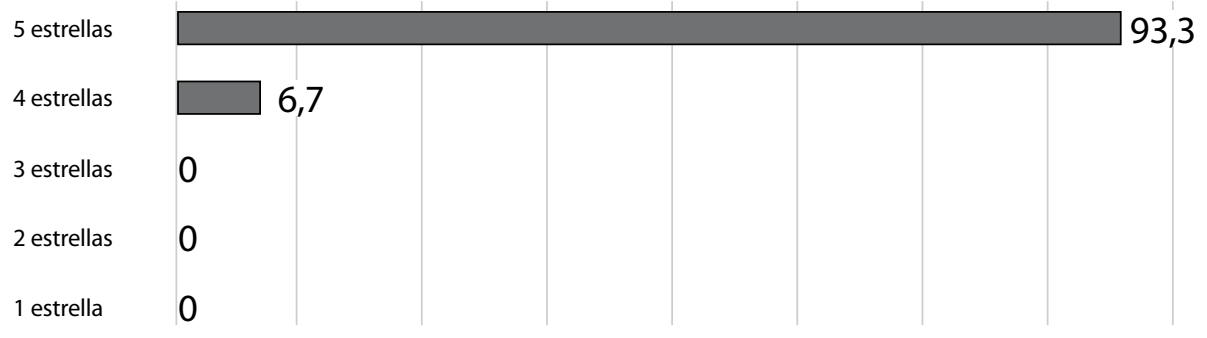


En la gráfica número 2, se puede observar que un $66,7 \%$ de los encuestados califica de cinco estrellas el precio en base al servicio, demostrando que el producto fue de alta calidad. Sin embargo, los encuestados comentaron lo siguiente: «Deberían valorarlo en mínimo dos dólares, por todo el servicio ofertado», dando a entender que estarían dispuestos a pagar hasta 7 veces más que su costo. Éste se debe al factor sorpresa que se les brindó, y cumpliendo sus expectativas, obtuvieron más de lo que esperaban.
En el siguiente gráficonúmero 3 núse demuestra que el buen servicio logró hacer sentir al 86,7\% de los encuestados como clientes valiosos, generando sentimientos de agrado y conformidad. Por otro lado, el 13,3\% posicionó el servicio como un producto de 4 estrellas. Lo más importante que se puede destacar es que todos los clientes vivieron un momento de atención y exclusividad. El sentimiento que arrojaron los comentarios de la entrevista contiene expresiones como: «me hicieron sentir especial», «me atendieron con una sonrisa» y otras similares acotaciones.

\section{Gráfica 2 - Diseño de barras, representación porcentual del nivel de satisfacción de cada uno de los clientes}

Valoración del precio con respecto al servicio

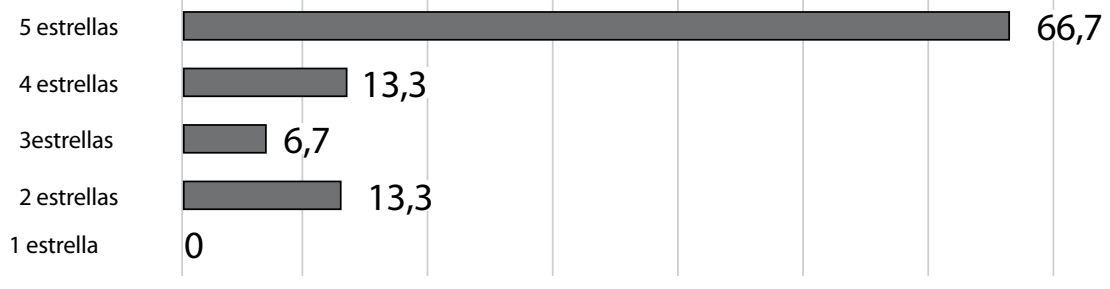

Gráfica 3 - Diseño de barras de la Valoración respecto al sentimiento de experiencia por parte de los clientes

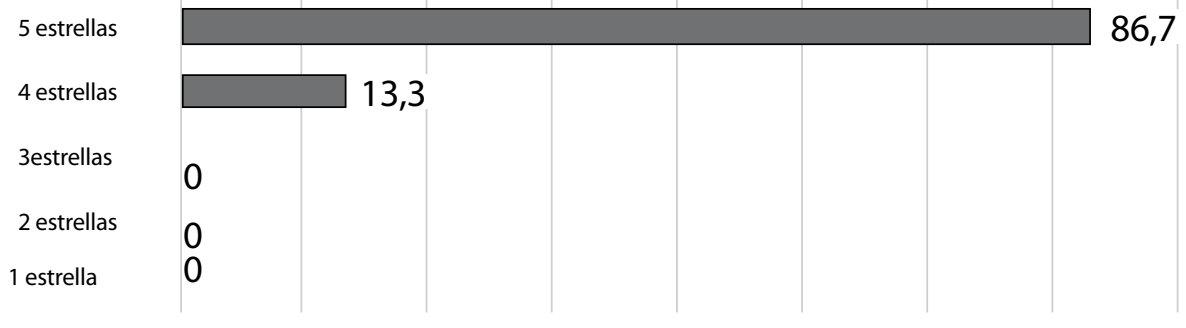




\section{Gráfica 4 - Diseño de Barras respecto a la calificación de una experiencia diferenciadora Calificación de la experiencia diferenciadora}

\begin{tabular}{|c|c|c|c|c|c|}
\hline \multicolumn{6}{|c|}{$7 \%$} \\
\hline & 1 estrella & 2 estrellas & 3 estrellas & 4 estrellas & 5 estrellas \\
\hline$\square$ Series 1 & $0 \%$ & $0 \%$ & $0 \%$ & $7 \%$ & $93 \%$ \\
\hline
\end{tabular}

La última pregunta realizada se direccionó a la experiencia del consumidor y estos fueron los resultados: el 93\% de los clientes considera que el servicio que recibieron fue una experiencia diferenciadora, dándole una calificación de 5 estrellas, y el 7\% lo catalogó con 4 estrellas.

\section{Conclusiones y discusión}

La comunicación efectiva conlleva la generación de una experiencia diferenciadora, marca un antes y después en el cliente. Es necesario recordar que existe un espacio para la duda entre la información de comunicación y la realidad que experimenta el usuario. El precio pasa a segundo plano e incluso, los componentes de un producto o servicio, cuando ya se experimenta la vivencia. Estamos en constante evolución y cada día, tenemos deseos aspiracionales ligados a los sentimientos propios o similares a los de nuestro entorno.

Crear una historia mediante una vivencia in situ cambia el panorama del servicio tradicional y la estimulación de los sentidos es clave en el proceso de compra. Los deseos, con frecuencia, son impulsivos y prevalecen sobre la racionalidad de un producto o servicio. El proceso de storytelling es clave para la puesta en escena de la propuesta de valor. Requiere dar un valor diferenciador que la competencia no dispone, en la actualidad es una forma efectiva de generar valor agregado real.

Para transmitir una experiencia es necesario tener presente que se necesitan dos elementos importantes: los módulos experienciales (MEE), que engloban las sensaciones, pensamientos, sentimientos, actuaciones y relaciones; y los proveedores de experiencia (ProvExs), que son los medios por los que se logra ejecutar, de forma controlada, los MEE (comunicación, identidad visual y verbal, presencia del producto, cogestión de marca, entornos espaciales, personal de la empresa, sitios web y otros medios electrónicos). El empleo adecuado y planificado de los MEE mediante los ProvExs conlleva cuidar cada uno de los aspectos de la experiencia.

Con todo, la valoración del precio se convierte en algo subjetivo. En necesario tener presente que no podemos medir con exactitud los gustos y preferencias de la audiencia en vista a que cada ser humano tiene patrones de conducta específicos. Crear valor a través de las acciones es un arte y requiere práctica para alcanzar destre- 
za mediante la experiencia. La creación de una experiencia satisfactoria incide directamente en el valor percibido y su vez influye directamente en la percepción sobre la vivencia (Tao, 2014). Que un cliente sienta felicidad después de haber pagado cinco veces más que el costo del producto realmente requiere estrategia, creatividad y humanidad. Hay necesidades humanas, en su mayoría de carácter sensorial y emocional que no se ven, pero están ahí. Es necesario desarrollar olfato creativo para detectarlas y saber cómo llegar a satisfacerlas mediante experiencias diferenciadoras que permanezcan no solo en la mente del cliente; es necesario llegar a su corazón.

Mediante la recopilación bibliográfica y la experimentación aplicada, se pudo comprobar que un usuario está dispuesto a pagar hasta cinco veces más por una experiencia diferenciadora. Así lo adelantaron las investigaciones realizadas por Bernd Schmitt desde los años 90 y hasta la actualidad. Otros autores confirman que los factores emocionales son importantes a la hora de tomar la decisión de compra en lo que se refiere a marcas y productos (García, Alcaraz y García, 2011). Fernández (2011) señala respecto a la gestión de intangibles: «Es necesario llegar al conocimiento de nuestros públicos objetivos, sus creencias y valores. Las campañas que llegan y conectan son aquellas que saben captar algún aspecto de nuestro target que le hace sentir empatía por nuestra oferta, más allá del mero ejercicio racional». Por ende, es necesario verificar la información publicitaria que se emite por medios de comunicación, debido a que impacta en la decisión de compra (Orden, Gómez y Paule, 2019).

Una de las principales ventajas de la presente investigación yace en la implementación de un servicio real para la comprobación de los objetivos de investigación. Se obtuvo de fuente directa de los usuarios sus apreciaciones y calificaciones respecto a su vivencia in situ. La elaboración de todo un storytelling vivencial ligado a la estimulación sensorial permitió generar un factor sorpresa en lo clientes que solicitaron su compra y generó el deseo de compra por los colaboradores de oficinas cercanas quienes emocionalmente hicieron pedidos en el momento. La valoración del precio respecto al servicio superó las expectativas ante los clientes. Sin embargo, las observaciones de la encuesta indicaron que los clientes estarían dispuestos a pagar hasta un $25 \%$ más por la misma atención. El factor WOW del servicio lo ponderaron sobre 5 estrellas y la calificación más baja fue 4 estrellas. La satisfacción en general estuvo en el 93\%.

La presente investigación no está exenta de limitaciones. Los resultados no son generalizables por la naturaleza del sector aplicado, se recomienda ampliar la muestra a otros servicios. Este trabajo apertura una nueva línea de investigación respecto a cómo la experiencia del consumidor influye en la fijación de precios en el sector de la restauración. 


\section{Bibliografía}

Anninou, I. and Foxall, G. (2019). The reinforcing and aversive consequences of customer experience. The role of consumer confusión. Journal of retailing and consumer services. 51, 139-151. doi: 10.1016/j.jretconser.2019.05.029

Avendaño, W., Paz, L. y Rueda, G. (2015). Estímulos auditivos en prácticas de neuromarketing. Caso: Centro Comercial Unicentro, Cúcuta. Cuadernos de Administración de la Universidad del Valle. 31 (53), 117-129. Cali, Colombia

Balan, F. (2015). Marketing Experiencial. Universal Miguel Hernández de Elche. 1-91.

Benavides, J. (2017). La gestión de las marcas y los valores en el turbulento contexto de la comunicación. Revista Internacional de Investigación en Comunicación aDResearch. 16 (16), 34 - 59. doi: org/10.7263/adresic-016-002

Castañeda, J., Del Valle, A. y Martínez, R. (2018). The effect of online and offline experiential marketing on brand equity in the hotel sector. Spanish Journal of Marketing. 22, 22-41. doi:org/10.1108/SJME-03-2018-003

Cuellar, S., Eyler, R. and Fanti, R. (2015). Experiential Marketing and Long-Term Sales. Journal of Travel and Tourism Marketing. 32(5), 534-553. doi: 10.1080/10548408.2014.918925

Erhardt, N., Martin, C y Chan, E. (2019). Value co-creation in sport entertainment between internal and external stakeholders. International Journal of contemporary hospitality management. 31 (11). doi: 10.1108/IJCHM-03-2018-0244

Fernández, J. (2011). Gestión de intangibles y brand density. Una aproximación teórica a la nueva estrategia de marca. Revista Internacional de Investigación en Comunicación aDResearch. 3 (3), 86 -103. doi: org/10.7263/adresic-003-02

Ferrés, J. \& Masanet, M. (2017). Communication Efficiency in Education: Increasing Emotions and Storytelling. Comunicar. 52, 51-60. doi: org/10.3916/C52-2017-05

Flores, J., Barrera, H. y Mayorga, M. (2019). Costos de producción y fijación de precios en empresas artesanales. Revista Publicando, 4 (12), 541-553.

García, M., Alcaraz, A. y García F. (2011). La influencia de las tecnologías de la información y comunicación en la distribución comercial en el pequeño comercio independiente. Revista Internacional de Investigación en Comunicación aDResearch. 3 (3), 106 a 123. doi:org/10.7263/adresic-003-01
Herrera, A. (2019). La disrupción exponencial de la comunicación digital y su impacto social. En Gedisa (Ed.), Comunicación persuasiva en las aulas: nuevas tendencias (193 -225). España, Barcelona: Editorial Gedisa.

Higueras, J., Alard, J. y Mercado, C. (2016) Las redes sociales y su efecto sobre la confianza en las tiendas online. Revista Internacional de Investigación en Comunicación. aDResearch. 14 (14), 30 - 53. doi: org/10.7263/adresic-014-04

Hoyer, W., MacInnis, D. y Pieters, R. (2015). Comportamiento del consumidor. México. Cengage Learning.

Juaneda, E., Olarte, C., y Pérez, M. (2018) Alternativas a la publicidad tradicional: Lovisual, un evento de éxito. Revista Internacional de Investigación en Comunicación aDResearch. 17 (17), 72 - 89. doi: org/10.7263/adresic-017-03

Jung, C. (2016). WOW Costumer Experience. Recuperado de: https://www.wowcx.com/que-es-experiencia-de-cliente/ Keiningham, T., Ball, J., Benoit (née Moeller), S., Bruce, H., Buoye, A., Dzenkovska, J., Nasr, L., Ou, Y. and Zaki, M. (2017), «The interplay of customer experience and commitment». Journal of Services Marketing. 31 (2), 148-160. doi: org/10.1108/JSM-09-2016-0337

La Experiencia del Consumidor. Cuando la marca conecta con las personas. (2017). Columna Brandstrategy \& Colors. Disponible en: https:/www.columnacolors.com/es/

Le,D., Scott, N. y Lohmann, G. (2019). Applying experiential marketing in selling tourism dreams. Journal of Travel and Tourism Marketing. 36 (2), 220-235. doi: 10.1080/1054 8408.2018.1526158

Lindstrom, M. (2015). Brand Sense- Building Powerful Brands Through Touch, Taste, Smell, Sight and Sound. New York, USA. Kogan Page Publishers. 53.

Llovet, C. (2018). The Role of Visual Merchandising to Position Fashion Retailers: a Key Place in Spanish Literature. Revista Internacional de Investigación en Comunicación aDResearch. 17 (17 ), 8 - 29. doi: org/10.7263/adresic-017-01

Majo, J. Moya, D y Vall-Llosera, L. (2018). Impacto de las redes sociales en los ingresos de los hoteles en Colombia, Ecuador y Perú. Revista Facultad de Ciencias Económicas: Investigación y Reflexión. 26 (1). doi: 10.18359/rfce.3143 Manzano, R., Gavilán, D., Avello, M., Abril, C., y Serra, T. (2012). Marketing sensorial, Comunicar con los sentidos en el punto de venta. Pearson Educación S.A. 69 - 129 
Nayeem, T., Murshed, F and Dwivedi, A (2019). Brand experience and brand attitude: examining a credibility-based mechanism. Marketing Intelligence and Planning. 37 (7), 821-836. doi: 10.1108/MIP-11-2018-0544

Orden, C., Gómez, R. y Paule, J. (2019) Sentimiento de los medios de comunicación españoles en formato digital sobre el Ibex 35. Revista Internacional de Investigación en Comunicación aDResearch. 19 (19), 56 - 67 doi:org/10.7263/adresic-019-03

Obiegbu, J., Larsen, G and Ellis, N. (2019). Experiential brand loyalty: towards an extended conceptualization of consumer allegiance to brands. Marketing theory. doi: org/10.1177/1470593119885167

Raiteri, M. (2016). El comportamiento del consumidor actual (Tesis de grado). Universidad Nacional de Cuyo, Mendoza.

Robles, M. (2009): La publicidad en el punto de venta: su realidad y tendencias. Revista de Comunicación. 18, 79 -128

Rodríguez, M. (2015). Política de fijación de precios: una nueva metodología basada en la estructura de costos-competencia de la empresa. Revista Internacional Administracion E Finanzas, 121.

Ruiz, A. (2019) Digital Fame and Fortune in the age of Social Media: A Classification of social media influencers. Revista Internacional de Investigación en Comunicación aDResearch. 19 (19), 8 - 29. doi:org/10.7263/adresic-019-01

Santana, L., Gómez, A. \& García, L. (2019). Uso problemático del móvil, fobia a sentirse excluido y comunicación familiar de los adolescentes. Comunicar. 59, 39-47. doi: org/10.3916/C59-2019-04

Schmitt, B. (1999). Experiental Marketing: How to Get Customers to Sense, Feel, Think, Act, and Relate to your Company and Brands. New York: Free Press.

Schmitt, B. (2000). Experiential Marketing Deusto. 1-17. Recuperado de https//preview.tinyurl.com/y4th986g

Schmitt, B. (2015). Experiental Marketing: A New Framework for Desing and Communications. DMI 40 Aniversarios. Edición 2015. 15, 19 - 26. Recovered from: https: // preview.tinyurl.com/yxexf3u7

Schmitt, B. H. (1999). Experiential Marketing. Journal of Marketing Management. 15 (1). United Kingdom.
Singhal, S. y Khare, K. (2015). Does Sense Reacts For Marketing-Sensory Marketing. International Journal of Management, IT and Engineering. 5 (5), 1-18.

Suarez, M., y García.G., (2012). Marketing sensorial, Cómo desarrollar la atmósfera del establecimiento comercial. Universidad Autónoma de Madrid. 1 - 12

Tao, S. (2014). Experiential Marketing and Marketing Experience: An Empirical Study of the Influence of Summer Boot Camp on Military Propensity. Military Psychology. 26, 5-6, 422-433. doi: 10.1037/mil0000059.

Uribe, M. (2017). Gerencia del servicio Alternativa para la competencia. Bogotá - Colombia: ediciones de la U.

Vidales, C. (2015). Historia, teoría e investigación de la comunicación. Comunicación y Sociedad. 23, 11-43. doi: org/10.32870/cys.v0i23.60

Victoria, I. and Herrero, A. (2020). The right to disconnect: An emerging right in the context of information and communication technologies. Izquierdas. (49), 1053-1074. Retrieved from www.scopus.com

Villanueva, H. y García, E. (2017). El olfato como refuerzo experiencial: aproximación cualitativa en el ámbito del marketing turístico. Revista Internacional de Investigación en Comunicación aDResearch. 54 - 71. doi: org/10.7263/adresic-015-03

Wisenblit, L. (2015). Comportamiento del consumidor Colombia Santa María la Ribera: Pearson

Wen, T., Qin, T and Liu, R. (2019). The impact of nostalgic emotion on brand trust and brand attachment an empirical study from China. Asia pacific journal of marketing and logistics. 31, 1118-1137. doi: 10.1108/APJML-09-2018-0390

Wu, H., Chen, X y Cheng, C. (2019). Relationships between experiential cultural distance, experiential relationship quality and experiential future intentions: the case of Mainland Chinese tourists. Journal of tourism and cultural change doi: 10.1080/14766825.2019.1679160

Zhang, H., Gordon, S., Buhalis, D., and Ding, X. (2018). Experience Value Co creation on Destination Online Platforms. Journal of Travel Research. 57(8), 1093-1107. https:// doi.org/10.1177/0047287517733557 